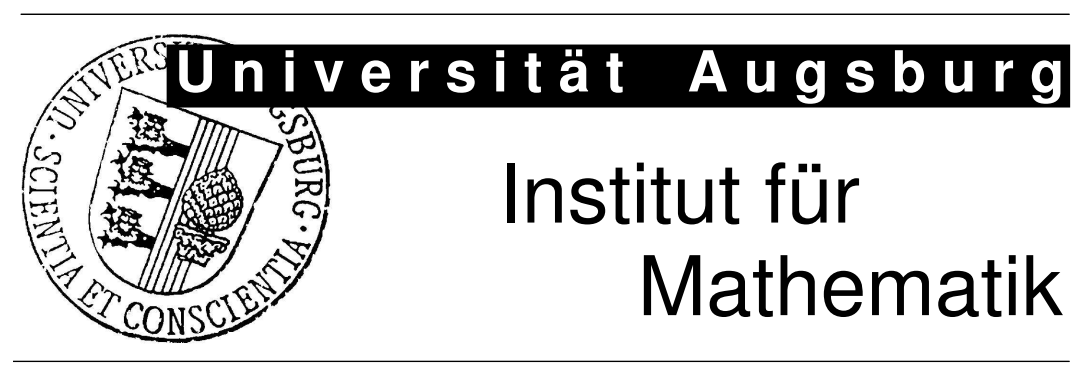

Dirk Blömker, Wael W. Mohammed, Christian Nolde, Franz Wöhrl Numerical Study of Amplitude Equations for SPDEs With Degenerate Forcing 


\section{Impressum:}

\section{Herausgeber:}

Institut für Mathematik

Universität Augsburg

86135 Augsburg

http://www.math.uni-augsburg.de/pages/de/forschung/preprints.shtml

\section{ViSdP:}

Dirk Blömker

Institut für Mathematik

Universität Augsburg

86135 Augsburg

Preprint: Sämtliche Rechte verbleiben den Autoren (C) 2011 


\title{
Numerical study of amplitude equations for SPDEs with degenerate forcing
}

\author{
Dirk Blömker, Wael W. Mohammed, Christian Nolde, Franz Wöhrl \\ Institut für Mathematik, Universität Augsburg, 86135 Augsburg
}

August 8, 2011

\begin{abstract}
We give a review on rigorous and numerical results for Amplitude equations. We focus on the Swift-Hohenberg equation and the Burgers equation, in order to see the quality of the approximation and the impact of degenerate noise on the approximating equation.
\end{abstract}

\section{Introduction}

Near a change of stability of a stochastic model, described by a stochastic partial differential equation (SPDE), one can use the natural separation of time scales, in order to derive approximating equations. These amplitude or modulation equations describe the behaviour of the amplitude of dominating pattern or modes. In the physics literature these equations are a successfully used to reduce the dynamics close to a bifurcation. See for [11] for a survey with many examples.

For deterministic partial differential equations (PDEs) in the last decades many rigorous results were established (see for example $[10,19,24,20,25,21]$ ) Here usually unbounded domains are considered, as on bounded domains center manifold theory is available, which is not established for SPDEs. For stochastic equations on bounded domains several results were established in recent years (see for example $[6,3,4]$ ). A first example on unbounded domains is [9].

In this paper we compare some rigorous analytic results with numerical simulations for the approximation of SPDEs. For simplicity of presentation we focus on two specific examples on an interval. One is an equation of Burgers type and the other the celebrated stochastic Swift Hohenberg equation. For the additive noise we consider for both examples either trivial degenerate forcing of a single Fourier mode or full space-time white noise.

In the introduction we first state our equations on the fast time-scale. Nevertheless, for the precise statement of results and the numerical simulations, we always consider the equations rescaled to the natural slow time-scale.

For the noise, in this work we consider only two examples for both equations. Either the equation exhibits a small space-time white noise forcing directly the dominant Fourier modes, or a highly degenerate noise acting only on a single non-dominant Fourier-Mode. In the second case one needs to consider much larger noise, as dominant modes are not forced and the noise needs nonlinear interaction in order to get mapped back to the dominant modes. 
Our example of Burgers type for a scalar $u(t, x) \in \mathbb{R}, t>0$ is

$$
\partial_{t} u=\left(\partial_{x}^{2}+1\right) u+\varepsilon^{2} \nu u-u \partial_{x} u+\sigma \xi_{\varepsilon},
$$

subject to Dirichlet boundary conditions for $x \in[0, \pi]$. The linear (in)stability $\varepsilon^{2} \nu u$ is chosen in a way that for solutions of order $\varepsilon$ both linear (in)stability and nonlinearity influence the dynamics of the dominating modes on the slow time-scale.

The constant $\sigma>0$ measures the noise strength relative to a prescribed power of $\varepsilon$. The noise term is either a one-dimensional noise acting only on the second Fourier mode

$$
\xi_{\varepsilon}(t, x)=\varepsilon \partial_{t} \beta(t) \sin (2 x),
$$

for some one-dimensional Brownian motion $\beta$, or

$$
\xi_{\varepsilon}(t, x)=\varepsilon^{2} \xi(t, x),
$$

where $\xi=\partial_{t} W$ is space-time white noise given by the formal derivative of a standard cylindrical Wiener process $W$ (cf. [12]).

The one-dimensional stochastic Swift-Hohenberg equation is given by

$$
\partial_{t} u=-\left(1+\partial_{x}^{2}\right)^{2} u+\varepsilon^{2} \nu u-u^{3}+\sigma \xi_{\varepsilon},
$$

subject to periodic boundary conditions for $x \in[0,2 \pi]$.

The noise term is either a spatially constant (or global) noise

$$
\xi_{\varepsilon}(t, x)=\varepsilon \partial_{t} \beta(t),
$$

for some Brownian motion $\beta$, or

$$
\xi_{\varepsilon}(t, x)=\varepsilon^{2} \xi(t, x),
$$

where $\xi$ is space-time white noise as before.

In both examples, the solution is well approximated by the dominant modes evolving on the slow time-scale $T=\varepsilon^{2} t$.

$$
u(t, x)=\varepsilon a\left(\varepsilon^{2} t\right) \cdot e(x)+\mathcal{O}\left(\varepsilon^{2}\right),
$$

where the amplitudes a satisfy an ordinary stochastic differential equation (SDE).

For the Burgers equation the amplitude $a(T) \in \mathbb{R}$ is one-dimensional, and the dominant mode is $e(x)=\sin (x)$. Moreover, in our two cases $a$ solves the following equations.

For space-time white-noise (cf. Blömker, Mohammed [7])

$$
\partial_{T} a=\nu a-\frac{1}{12} a^{3}+\sigma \partial_{T} \hat{\beta},
$$

where $\hat{\beta}$ is the rescaled direct impact of the noise on the dominant modes. It is a Brownian motion given by $\hat{\beta}(T) \sin =\varepsilon P_{c} \tilde{W}(T)$ with $P_{c}$ being the orthogonal projection on the sin and $\tilde{W}(T)=\varepsilon W\left(\varepsilon^{-2} T\right)$ a rescaled version of the Wiener process.

For degenerate noise given by (2) Blömker, Hairer \& Pavliotis [4] furnished that $a$ solves

$$
d a=\left(\nu-\frac{\sigma^{2}}{88}\right) a d t-\frac{1}{12} a^{3} d t+\frac{\sigma}{6} a \circ d \tilde{\beta},
$$


which is interpreted as a Stratonovic equation, where the noise is given by the rescaled original Brownian motion $\tilde{\beta}(T)=\varepsilon \beta\left(T \varepsilon^{-2}\right)$, with $\beta$ from (2) .

Note that the term $\frac{\sigma^{2}}{88} a$ is not only an Itô-Stratonovic correction. It contains additional terms that arise due to nonlinear interaction of the noise term with itself.

For the Swift-Hohenberg equation in (5) the amplitude $a(T) \in \mathbb{R}^{2}$ is twodimensional for the dominant modes $e(x)=(\sin (x), \cos (x))$.

For space-time white noise (cf. Blömker \& Hairer [2]) the amplitude solves

$$
\partial_{T} a=\nu a-\frac{3}{4} a|a|^{3}+\partial_{T} \hat{\beta},
$$

where $\hat{\beta}$ is a two dimensional Brownian motion, which is as in (6) the direct impact of the Wiener process $W$, which is rescaled to the slow time-scale and projected to the dominant modes.

Surprisingly, for global noise the amplitude $a \in \mathbb{R}^{2}$ solves a deterministic ODE (cf. Blömker \& Mohammed [8])

$$
\partial_{T} a=\left(\nu-\frac{3}{2} \sigma^{2}\right) a-\frac{3}{4} a|a|^{3} .
$$

Again, noise feeds back into the dominant modes via nonlinear interaction. Note that, obviously, the constant $\frac{3}{4}$ depends on the normalization of the dominant mode.

For the numerical simulation in order to compare the SPDE with the amplitude equation, we implemented a straightforward semi-implicit timediscretisation of a spectral Galerkin method using fast Fourier transforms. It turned out that a few modes are enough to give an accurate description of the dynamics (see also [1]). Nevertheless in most cases we used 128 Fourier-Modes for spacial discretization. For the time-discretisation, for simplicity of implementation, we used a constant small time-step, lie for example $h=10^{-6}$. More details on the method will be given in Section 2.2 for the Burgers equation only.

See the work of Jentzen et.al. (see $[17,18]$ ) for rigorous results and methods with improved order of convergence. Moreover, [5] give a result on the approximation of Burgers equation with spectral Galerkin method using uniform topology.

The numerical simulations were mainly done by Wöhrl and Nolde in their Diploma thesis. They studied the quality of the theoretical result. It turned out, that the approximation is valid on very long time-scales even for moderate values of $\varepsilon$ like $\frac{1}{10}$ or $\frac{1}{100}$, while the theoretical result predicts that the approximation is valid on some fixed time-interval in the limit $\varepsilon \rightarrow 0$.

Due to the stiffness of the equations $\varepsilon \rightarrow 0$ is obviously not accessible with direct numerical simulations of the SPDEs. It is well-known that semi-implicit methods are stable, but due to linear damping only one or two modes are effectively calculated, if $\varepsilon$ is too small. Here more advances multi-scale methods need to be considered (see for example [1] or [13]), but these methods rely on the multi-scale approximation results, which we wanted compare to the numerical simulation of the SPDE.

Moreover, using the numerical simulations we tried to find higher order effects not given by the amplitude equation, which might be useful to understand the main sources of errors in the approximation.

In many cases, the numerical error estimate is much better than expected. Here various other small terms or large eigenvalues improved the error bounds. 
For example the 10th eigenvalue of the Swift-Hohenberg operator is -81 , which is (compared to $\varepsilon=1 / 10$ ) already of order $\varepsilon^{-2}$. Moreover, a small $\sigma$ improves the estimates, too (cf. Figure 12). Nevertheless, for the theorems all these terms are treated as order $\mathcal{O}(1)$ in $\varepsilon$.

In the following Section 2 we discuss first the Burgers type equation. While Section 2.1 states the theorems, Section 2.2 gives numerical results. Section 3 provides the results for Swift-Hohenberg with theorems in Section 3.1 and numerics in Section 3.2. In the final Section 4, we discuss large (or unbounded) domains, which are from a computational point even worse.

\section{Burgers equation}

For the precise statement of results, we always rescale the equations to the slow time-scale $T=\varepsilon^{2} t$. The rescaled Burgers equation for $v$, where $u(t, x)=$ $\varepsilon v\left(\varepsilon^{2} t, x\right)$ reads

$$
\partial_{t} v=\frac{1}{\varepsilon^{2}}\left(\partial_{x}^{2}+1\right) v+\nu v-\frac{1}{\varepsilon} v \partial_{x} v+\sigma \xi_{\varepsilon}
$$

subject to Dirichlet boundary conditions for $x \in[0, \pi]$.

We consider two extreme cases of noise. First, the noise term is either a highly degenerate one-dimensional noise acting only on the second Fourier mode, which is not dominant:

$$
\xi_{\varepsilon}(T, x)=\frac{1}{\varepsilon} \partial_{T} \tilde{\beta}(T) \sin (2 x)
$$

for a single Brownian motion denoted by $\tilde{\beta}$. Secondly, we consider space-time white noise $\xi_{\varepsilon}=\partial_{T} \tilde{W}$ given by a formal derivative of a standard cylindrical Wiener process $\tilde{W}$ (cf. [12]).

Denote by $P_{c}$ the orthogonal projection onto the dominant space $\mathcal{N}=$ $\operatorname{span}\{\sin \}$ and let $P_{s}=I-P_{c}$ be the orthogonal projection onto the other modes.

\section{$2.1 \quad$ Rigorous result}

Let us state the rigorously known approximation results for the Burgers equation. For simplicity of presentation, we restrict to the case of our examples presented here. Most of the theorems are proven in a more general setting. For space-time white noise [7] provided:

Theorem 1. (Approximation - full noise) Let $v$ be a continuous solution of (10) in $H^{\alpha}([0, \pi]), \alpha \in\left[0, \frac{1}{2}\right)$, with initial condition $v(0)=a(0) \sin +\varepsilon \psi(0)$ where $a(0)$ and $\psi(0) \perp \sin$ are of order one. Suppose that a is a solution of the amplitude equation (6). Then for all $p>1, \kappa>0$ and $T_{0}>0$ there exists a constant $C>0$ such that

$$
\mathbb{P}\left(\sup _{T \in\left[0, T_{0}\right]}\|v(T)-a(T) \sin \|_{H^{\alpha}}>\varepsilon^{1-\kappa}\right) \leq C \varepsilon^{p}
$$

for all $\varepsilon>0$ sufficiently small.

Note that for short-hand notation (12) in the previous statement of the theorem, is sometimes abbreviated as $v(T)=a(T)+\mathcal{O}\left(\varepsilon^{1-}\right)$. Moreover, $H^{\alpha}([0, \pi])$ 
denotes the standard fractional Sobolev space. Later in the numerics, we only consider the uniform topology using $L^{\infty}([0, \pi])$-norms.

For degenerate noise, if the noise is not acting directly on the dominant modes, by Theorem 1 the noise disappears in the amplitude equation. Thus in that case we discuss noise that is an order of magnitude larger. Now via nonlinear interaction the noise has the potential to reappear in the amplitude equation. This was for the Burgers equation first observed by Roberts [22] and later proven rigorously by Blömker, Hairer, Pavliotis [4].

Here we only consider the possibly most simple case of degenerate noise given by (11). Nevertheless, as in [4] we could also force all modes except the dominant ones. In that case the coefficients in the amplitude equation will turn into series due to infinitely many nonlinear interactions. Moreover, the amplitude equation would contain multiplicative as well as additive noise.

Theorem 2. (Approximation - degenerate noise) Let $u$ be a continuous $H_{0}^{1}([0, \pi])$-valued solution of (10) with degenerate noise and with initial condition $u(0)=\mathcal{O}(\varepsilon)$. If a is a solution of (7) with $a(0) \sin =P_{c} u(0)$ and

$$
R(T)=\frac{1}{\varepsilon} e^{-L T \varepsilon^{-2}} P_{s} u(0)+\frac{1}{\varepsilon} \int_{0}^{T} e^{-3(T-s) \varepsilon^{-2}} d \tilde{\beta}(s) \sin (2 \cdot),
$$

then for all $\kappa, T_{0}, p>0$ there is a constant $C$ such that

$$
\mathbb{P}\left(\sup _{T \in\left[0, T_{0}\right]}\|v(T)-a(T) \sin -R(T)\|_{H^{1}}^{p}>\varepsilon^{\frac{1}{2}-\kappa}\right) \leq C \varepsilon^{p} .
$$

It is not proven, but the previous result should hold with errors of order $\mathcal{O}\left(\varepsilon^{1-}\right)$ instead of $\mathcal{O}\left(\varepsilon^{\frac{1}{2}-}\right)$.

Let us discuss the additional term $R$ in more detail. The dependence on the initial condition dies out very fast. This was not included in Theorem 1, but it can easily be added there, too.

As $f_{\varepsilon}(s)=\varepsilon^{-2} e^{-(t-s) \varepsilon^{-2}}, s \in[0, t]$, converges for $\varepsilon \rightarrow 0$ to a Deltadistribution in $t$, the stochastic integral behaves like a white noise of order $\mathcal{O}(\varepsilon)$ acting on the second Fourier mode. Thus formally it is a term of higher order, although it can easily be shown that the stochastic convolution itself is almost order one, i.e. $\mathcal{O}\left(\varepsilon^{0-}\right)$.

We will discuss some ideas of the proofs later in the Section 3.1 on SwiftHohenberg. In the following section we discuss our numerical results for the Burgers equation.

\subsection{Numerical result}

For our simulation we use a semi-implicit Euler method. To illustrate this consider a PDE with linear operator $A$ :

$$
\partial_{T} u(T)=A u(T)+f(u(T), T) .
$$

We fix a constant step-size $h$ and approximate:

$$
u(T+h) \approx \underbrace{[1-h A]^{-1}}_{:=M}[u(T)+h f(u(T), T)]=M[u(T)+h f(u(T), T)] .
$$


The linear operator $M$ of this semi-implicit Euler method applied to our equation (10) is in Fourier space a diagonal matrix with entries

$$
M_{k}=\left[1-h\left(\varepsilon^{-2}\left(1-k^{2}\right)+\nu\right)\right]^{-1} .
$$

Thus $M_{k} \leq 1$ for all $k \in \mathbb{N}, M_{k}$ is strictly decreasing for $k$ to infinity, and $M_{k} \stackrel{k \rightarrow \infty}{\longrightarrow} 0$. Nevertheless, it should not be too small, in order that sufficiently many modes are effectively calculated. So the operator $M$ is damping all modes except the first $\left(M_{1}=1\right)$, and it is sufficient to observe the first few (depending on $h$ and $\varepsilon$ ) Fourier modes because the higher modes converge very fast to zero.

For the spacial discretisation we implemented in Matlab a spectral Galerkin method with $N=127$ Fourier modes. Note that $2^{n}-1$ modes are optimal for the fast discrete sine and cosine transformation used to implement the nonlinearity.

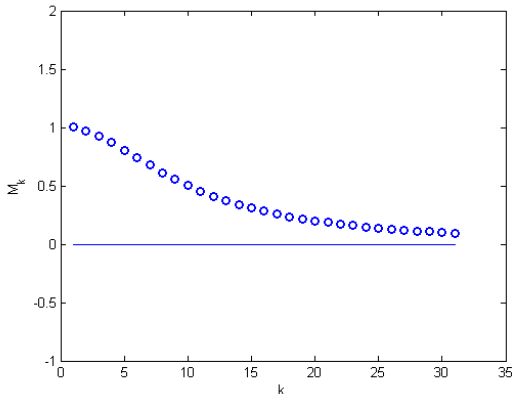

(a) $\varepsilon=0.1$

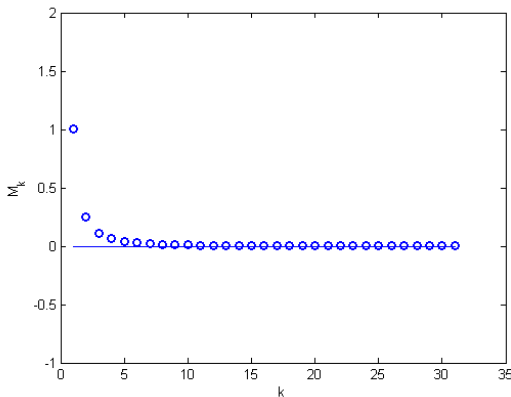

(b) $\varepsilon=0.01$

Figure 1: Values of the linear operator $M$ for different $\varepsilon$. Parameters: $\nu=0.5$, $h=0.0001, N=31$.

In addition to the amplitude equation given by (6), we introduce two heuristic terms in order to improve our approximations in the case of white noise. Those terms and their respective approximation error is defined by

$$
\begin{aligned}
& R_{1}(T)=\sup _{x \in[0, \pi]}\|u(T, x)-a(T) \sin (x)\|, \\
& R_{2}(T)=\sup _{x \in[0, \pi]}\left\|u(T, x)-a(T) \sin (x)+\varepsilon \frac{1}{6} a^{2} \sin (2 x)\right\|,
\end{aligned}
$$

and

$$
R_{3}(T)=\sup _{x \in[0, \pi]}\left\|u(T, x)-a(T) \sin (x)+\varepsilon \frac{1}{6} a^{2} \sin (2 x)-O(T)\right\|,
$$

where $a$ is the solution of the amplitude equation on $\left[0, T_{0}\right]$ and $O$ is an OrnsteinUhlenbeck process given by

$$
\partial_{T} O(T)=\varepsilon^{-2}\left(1+\partial_{x}^{2}\right) O(T)+\sigma \sum_{k=2}^{N} \beta_{k}^{\prime}(T) \sin (k x) .
$$




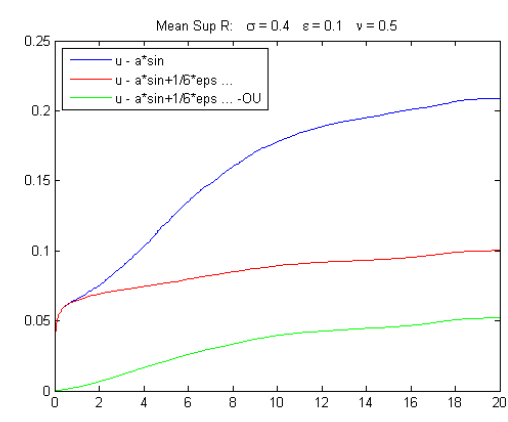

(a) mean $\sup R, 300$ realisations, $\varepsilon=0.1$

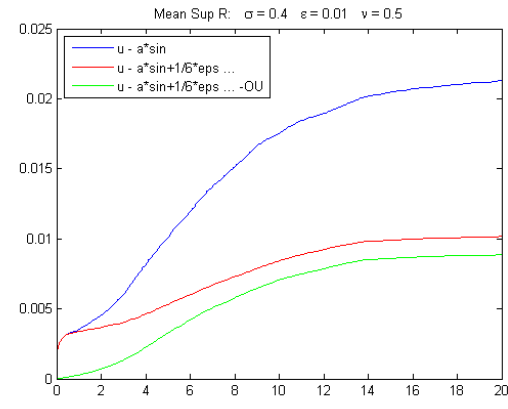

(b) mean $\sup R, 100$ realisations, $\varepsilon=0.01$

Figure 2: The mean $\sup R_{i}$ for white noise and $i=1,2,3$ for parameters $\sigma=0.4$, $\nu=0.5, u_{0}=0$. Note that $R_{1}>R_{2}>R_{3}$.

Those errors are expected to hold

$$
R_{1}(T) \approx \mathcal{O}(\varepsilon), \quad R_{3}(T) \approx \mathcal{O}\left(\varepsilon^{2}\right), \quad R_{1}(T) \geq R_{2}(T) \geq R_{3}(T) .
$$

While this is proven for $R_{1}$ in Theorem 1 , the estimates for $R_{2}, R_{3}$ are heuristic.

In the case of degenerated noise, we have the approximation result of Theorem 2 with error

$$
R_{4}(T)=\sup _{x \in[0, \pi]}\|u(T, x)-a(T) \sin (x)-Z(T) \sin (2 x)\| .
$$

of order $R_{4}=\mathcal{O}\left(\varepsilon^{\frac{1}{2}-}\right)$. Here we denote by $Z$ the OU-process contained in $R$ from Theorem 2 .

In order to approximate $\mathbb{E} \sup _{[0, T]} R_{i}$, we use the standard Monte-Carlo method and simulate different realisations. For $S$ independent realisations we define

$$
\text { mean sup } R_{k}(T)=\frac{1}{S} \sum_{i=1}^{S} \sup _{t \in[0, T]}\left\{R_{k}^{(i)}(t)\right\} \quad \text { for } \quad k \in\{1,2,3,4\}
$$

For white noise we typically obtain results as in Figure 2. They show that the expected orders for $R_{i}, i \in\{1,2,3\}$ hold for quite large times, and that the heuristic terms yield significant improvements.

For white noise and unstable $\nu>0$, we identified a specific source of errors. If both the amplitude $a(t)$ and the first Fourier mode $u^{(1)}(t)$ are close to zero, it can happen that they "split" up and, in worst case, converge to different critical points. An example is presented in Figure 3. We conjecture that these trajectories are responsible for most of the error, although they are rare events. 


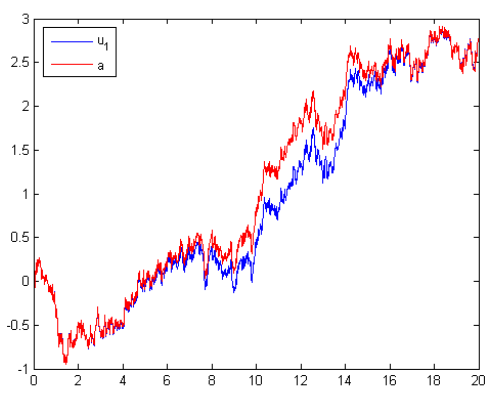

(a) $u^{(1)}(t)$ and $a(t)$ split up and reunite.

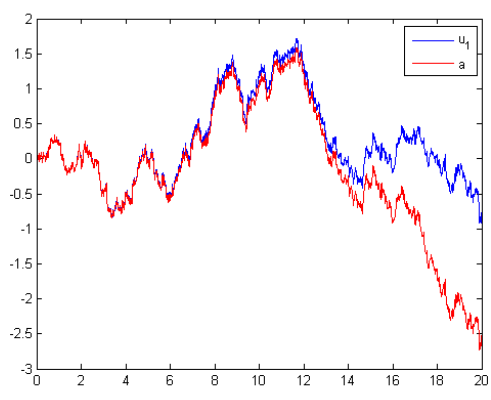

(b) $u^{(1)}(t)$ and $a(t)$ split up.

Figure 3: The first Fourier mode $u^{(1)}(t)$ and the amplitude $a(t)$ split near zero for white noise. Parameters: $\nu=0.5, \varepsilon=0.1, \sigma=0.4, u_{0}=0$.

Sadly, among a few thousand simulations we never found a realisation splitting up to and converging to the two different critical points.

For degenerated noise we obtain results for $R_{4}(t)$ as in Figure 4.

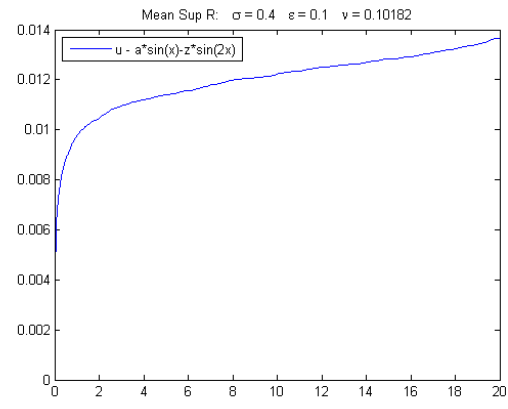

(a) mean sup $R_{4}, 300$ realisations, $\varepsilon=0.1$

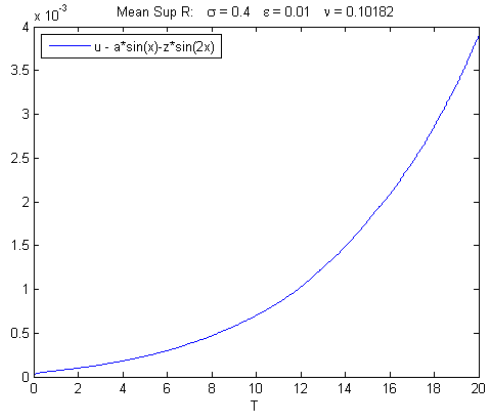

(b) mean $\sup R_{4}, 100$ realisations, $\varepsilon=0.01$

Figure 4: mean $\sup R_{4}$ for degenerate noise with parameters: $\sigma=0.4, \nu=$ $\frac{\sigma^{2}}{88}+0.1, u_{0}=0.01 \sin (x)$.

The splitting up as in the case of white noise can not happen for degenerated noise because $u^{(1)}(t)$ and $a(t)$ are not able to change their sign. The amplitude equation is obviously not able to change the sign due to the multiplicative noise, and the deterministic fixed point 0 . From numerical simulations with quite small $\nu>0$ (cf. Figure 5) there is a strong indication that this holds true for $u^{(1)}$, too. The limitation to 12 realisations in the Figure is just for presentational purpose. Among a thousand of realisations with varying parameters none changed the sign.

The last aspect we study is the fact that in Theorem 1 and Theorem 2 the estimates are only valid for some fixed (possibly very small) $T_{0}$. So in our experiments we took a look on how large this $T_{0}$ might be. 


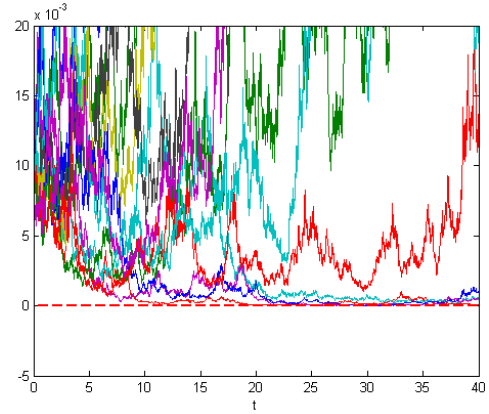
$\begin{array}{ll}\text { (a) } u_{0}=0.01 \sin (x), \sigma=3, \nu=\frac{\sigma^{2}}{88}+0.001, & \text { (b) } u_{0}=0.01 \sin (x), \sigma=5, \nu=-0.001, \\ \varepsilon=0.01 & \varepsilon=0.1\end{array}$

Figure 5: Typical 12 realisations each of $u^{(1)}$ with degenerate noise. The Fourier mode $u^{(1)}$ is not changing its sign.

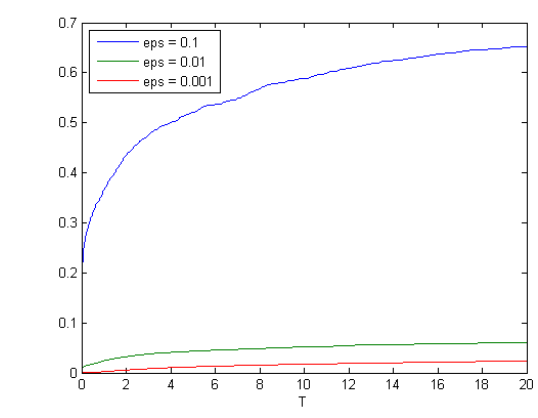

(a) mean $\sup R_{1}$

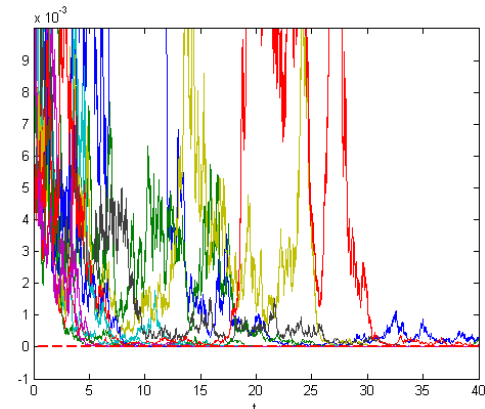

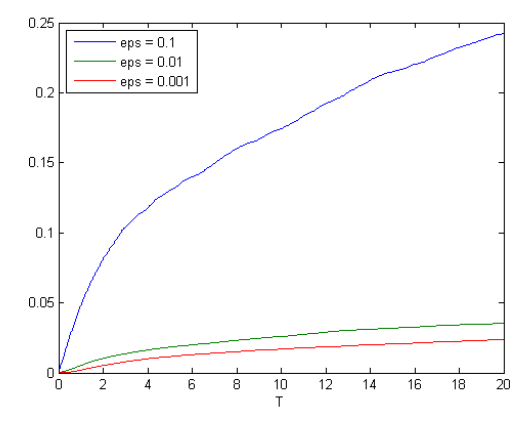

(b) mean $\sup R_{3}$

Figure 6: mean $\sup R_{i}$ for different $\varepsilon$ with white noise and 200 realisations for each $\varepsilon$. Parameters: $\sigma=2, \nu=0.5, u_{0}=0.01 \sin (x)$.

Define

$$
T_{\max }:=\min _{T}\left\{\operatorname{mean} \sup R_{i}(T)>K_{i}\right\}
$$

and

$$
\tilde{T}_{\max }:=\min _{T}\left\{\operatorname{mean} \sup R_{i}(T)>10 K_{i}\right\}
$$

in order to decide whether $R_{i}$ is $\mathcal{O}\left(K_{i}\right)$ or not. As stated before, $K_{1}=K_{2}=\varepsilon$, $K_{3}=\varepsilon^{2}$ and $K_{4}=\sqrt{\varepsilon}$. For white noise it turned out that this is true for quite large $T_{0}$, but it highly depends on $\varepsilon$ (see Figure 6 and 7 ). The results are not always that good for other parameter combinations, but even $T_{\max }=2$ is a reasonable result. Moreover, for very small $\varepsilon$ like 0.001 , we run into the problem, that the step-size $h$ might be too large in the simulation.

For degenerated noise the results can be seen in Figure 8. As for white noise, the estimate holds for relative large $T_{0}$, and we need to be careful, if $\varepsilon$ is too small. 


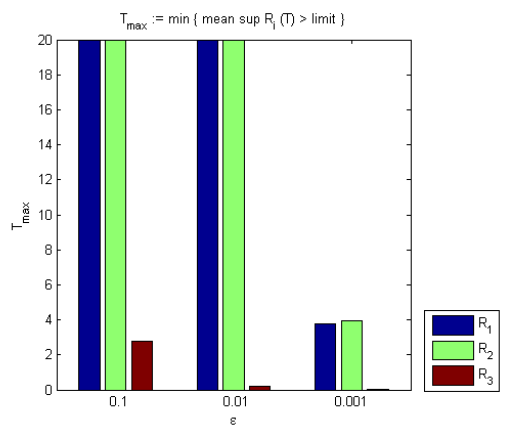

(a) $T_{\max }:=\min _{T}\left\{\right.$ mean $\left.\sup R_{i}(T)>K_{i}\right\}$

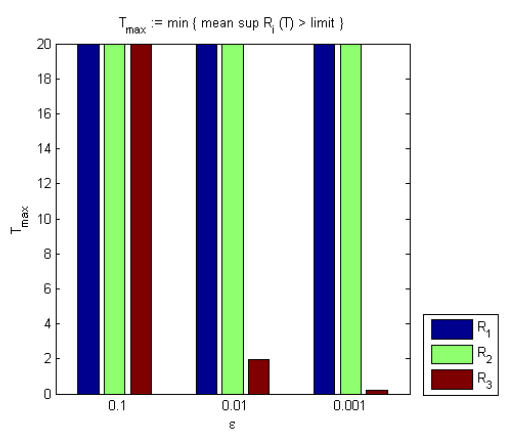

(b) $T_{\max }:=\min _{T}\left\{\operatorname{mean} \sup R_{i}(T)>10 K_{i}\right\}$

Figure 7: Maximal $T_{\max }$ for mean $\sup R_{i}$ for different $\varepsilon$ with white noise and 200 realisations for each $\varepsilon$. Parameters: $\sigma=2, \nu=0.5, u_{0}=0.01 \sin (x)$.

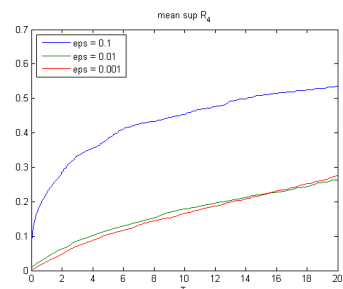

(a) mean $\sup R_{4}$

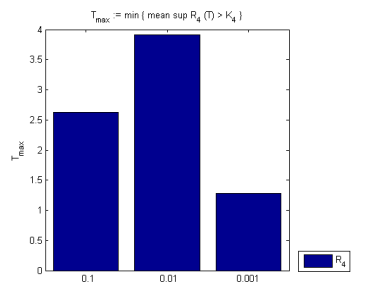

(b) $T_{\max }:=\min _{T}$ $\left\{\right.$ mean $\left.\sup R_{4}(T)>\sqrt{\varepsilon}\right\}$

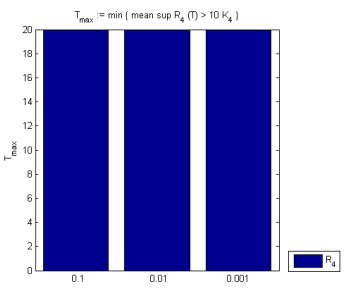

(c) $T_{\max }:=\min _{T}$ $\left\{\right.$ mean $\left.\sup R_{4}(T)>10 \sqrt{\varepsilon}\right\}$

Figure 8: mean $\sup R_{4}$ for different $\varepsilon$ with degenerate noise and 200 realisations for each $\varepsilon$. Parameters: $\sigma=4, \nu=\frac{\sigma^{2}}{88}+0.1, u_{0}=0.8 \sin (x) . h$ dependent on $\varepsilon\left(h=10^{-4}\right.$ for $\varepsilon=0.1,0.01$ and $h=10^{-6}$ for $\left.\varepsilon=0.001\right)$ and $N=31$ (to compensate the smaller stepsize with regard to computation time).

\section{Swift-Hohenberg equation}

Let us first state the rescaled equation on the slow time-scale again with our two very different cases of noise. The rescaled stochastic Swift-Hohenberg equation for $v$, where $u(t, x)=\varepsilon v\left(\varepsilon^{2} t, x\right)$ reads

$$
\partial_{t} v=-\frac{1}{\varepsilon^{2}}\left(1+\partial_{x}^{2}\right)^{2} v+\nu v-v^{3}+\xi_{\varepsilon}
$$

subject to periodic boundary conditions for $x \in[0,2 \pi]$. We denote the linear operator by $L=-\left(1+\partial_{x}^{2}\right)^{2}$.

We consider again two extreme cases of noise. First, the noise term is a onedimensional noise acting on the constant Fourier mode, which is not dominant:

$$
\xi_{\varepsilon}(T, x)=\frac{1}{\varepsilon} \partial_{T} \tilde{\beta}(T)
$$

for a real-valued Brownian motion $\tilde{\beta}$. Secondly, $\xi_{\varepsilon}=\partial_{T} \tilde{W}$ is space-time white noise. 
Now the dominant space is $\mathcal{N}=\operatorname{span}\{\sin , \cos \}$, and we denote as in the case of Burgers equation the $L^{2}$-orthogonal projection onto it by $P_{c}$, while the projection onto the orthogonal complement $\mathcal{S}$ is given by $P_{s}$. Note that $P_{c}$ is different for (10) and (13) as the dominant space $\mathcal{N}$ has different dimensions.

\subsection{Rigorous result}

For space-time white noise Blömker \& Hairer [2] provided an approximation result including higher order corrections. This is in this case just a fast OrnsteinUhlenbeck-process on $\mathcal{S}$. Moreover, this approximation carries over to an invariant measure of (13), see [2]. But here we focus on the transient approximation result, only.

In order to define the approximation split

$$
\psi=\psi_{c}+\varepsilon \psi_{s} \quad \text { with } \quad \psi_{c}(T):=a(T) \cdot(\sin , \cos ),
$$

and define $\psi_{s}$ by $\psi_{s}(0)=\varepsilon^{-1} P_{s} v_{0}$ and

$$
\psi_{s}(T)=e^{T L \varepsilon^{-2}} \psi_{s}(0)+P_{s} \tilde{W}_{L}(T)
$$

where the stochastic convolution $\tilde{W}_{L}$ is defined as

$$
\tilde{W}(T)=\frac{1}{\varepsilon} \int_{0}^{T} e^{(T-s) L \varepsilon^{-2}} d \tilde{W}(s),
$$

which is an $\varepsilon$ independent Ornstein-Uhlenbeck process on the fast time-scale $t$.

For the initial conditions we assume

$$
\mathbb{E}\left\|v_{0}\right\|^{p} \leq C_{p} \text { and } \quad \mathbb{E}\left\|P_{s} v_{0}\right\|^{p} \leq C_{p} \varepsilon^{p}
$$

Theorem 3. (Approximation - full noise) Let $v$ be the mild solution in $C^{0}\left([0, \infty), L^{2}\right)$, of (13) with initial value $v_{0}$ of order $\mathcal{O}(1)$ being $\mathcal{O}(\varepsilon)$ on the stable modes, for instance satisfying (16). Define the approximation $\psi$ as in (14) with a given by the amplitude equation (6).

Then for all $p>0, \kappa>0$ and $T_{0}>0$ there is a constant $C>0$ explicitly depending on $p$ and growing exponentially with $T_{0}$ such that the estimate

$$
\mathbb{P}\left(\sup _{T \in\left[0, T_{0}\right]}\|v(T)-\psi(T)\|_{L^{2}} \leq \varepsilon^{2-\kappa}\right) \geq 1-C \varepsilon^{p}
$$

holds for $\varepsilon>0$ sufficiently small.

It can be justified in a very general setting, that starting with initial conditions $v_{0}$ of order $\mathcal{O}(1),(16)$ holds after a very short time of order $\mathcal{O}\left(\varepsilon^{2} \ln (\varepsilon)\right)$. This is based on linear stability only and called attractivity. Nevertheless, for Swift-Hohenberg this result is significantly better. Due to nonlinear stability the solution for any initial condition satisfies (16) after any small time of order 1 .

For degenerate noise Blömker \& Mohammed [8] showed:

Theorem 4. (Approximation - degenerate noise) Let $v$ be a continuous solution of (13) in $H^{\alpha}, \alpha \geq 0$, with degenerate noise. Suppose for the initial 
conditions $v(0)=a(0) \cdot(\sin , \cos )+\psi(0)$, where $\psi(0) \in \mathcal{S}$, and $a$ is a solution of the amplitude equation (9).

Then for all $p \geq 1, T_{0}>0$ and all $\kappa>0$ sufficiently small, there exists $C>0$ such that for $\|v(0)\|_{\alpha} \leq \delta_{\varepsilon}$ for some $\delta_{\varepsilon} \in\left(0, \varepsilon^{-\frac{1}{3} \kappa}\right)$ we have

$$
\mathbb{P}\left(\sup _{T \in\left[0, T_{0}\right]}\|v(T)-a(T) \cdot(\sin , \cos )-\mathcal{Q}(T)\|_{\alpha}>\varepsilon^{1-\kappa}\right) \leq C \varepsilon^{p},
$$

where

$$
\mathcal{Q}(T)=e^{\varepsilon^{-2} T L} \psi(0)+\mathcal{Z}(T)
$$

with a fast $O U$-process

$$
\mathcal{Z}(T)=\sigma \varepsilon^{-1} \int_{0}^{T} e^{-\varepsilon^{-2}(T-\tau)} d \beta(\tau) .
$$

For the proof of the result in both cases, one first shows that $v$ is bounded by $\mathcal{O}(1)$, using standard energy type estimates. Secondly, one improves the bound for $P_{s} v$, which is approximated by (15) or (18) using the mild formulation. In the third step, one identifies all small terms from the equation for $P_{c} v$, which defines the residual. This step needs explicit averaging over the fast OU-process with explicit error bounds, that are established using Itô-formula. In the final step, one removes the residual from the equation for $P_{c} v$, by approximation arguments for ODEs, which yields the amplitude equation.

For Swift-Hohenberg one can rely on nonlinear stability, but for Burgers equation it is helpful to work with a stopping time ensuring that $v$ is order one up to the stopping time. Then all estimates are done up to the stopping time, and finally the amplitude equation is used to show that the stopping time is of order one.

\subsection{Numerical result}

For the Swift-Hohenberg equation we used the analogous methods as for Burgers and implemented the spectral Galerkin approximation in Matlab using 128 Fourier modes. For the time-discretisation we used again for simplicity a semiimplicit method with constant step-size of order $h=10^{-6}$, which seems to be sufficient for $\varepsilon=0.1$ and $\varepsilon=0.01$.

Let us give here in the degenerate noise case an estimate of the mean error

$$
R(T)=\mathbb{E}\|v(T)-a(T) \cdot(\sin , \cos )-\mathcal{Z}(T)\|_{\infty}
$$

and the mean uniform error

$$
R_{\text {sup }}(T)=\mathbb{E} \sup _{s \in[0, T]}\|v(s)-a(s) \cdot(\sin , \cos )-\mathcal{Z}(s)\|_{\infty} .
$$

We used sufficiently small deterministic initial conditions. In Figure 9 the parameters are $\nu=1$ and $\sigma=1 / \sqrt{15}$, which is a very small noise. It turned out that this improves the numerical bound on the error a lot.

The numerical result shows the excellent quality of the approximation. After an initial phase, the mean error seems to be uniformly small for large times, while the mean uniform error grows very slowly over time. 

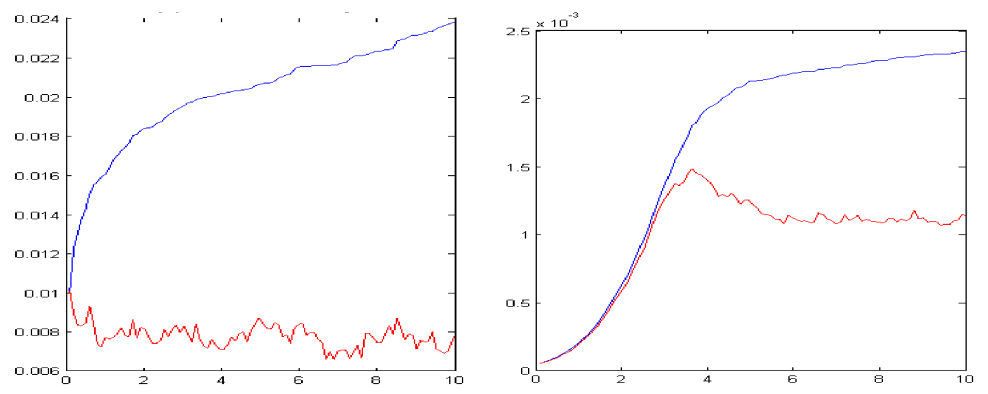

Figure 9: Mean error over a few hundred realisations for degenerate noise. Parameters: $\nu=1, \sigma=1 / \sqrt{15}$ On the left: $\varepsilon=0.1$. On the right: $\varepsilon=0.01$. Small initial conditions of order $\varepsilon$.

We can use various values of $\nu, \varepsilon, \sigma$ and $T$. In all cases the numerical error bound looks very similar. Nevertheless, due to the smallness of $\sigma$, which is of the order of $\mathcal{O}(\varepsilon)$, the error bound seems to be much better than $\mathcal{O}(\varepsilon)$. If we increase $\sigma$ the error will increase, too. See also Figure 12, later.

In Figure 10 we see that even over a very long time the error does not increase much. Again, the small $\sigma$ makes the bound much better.

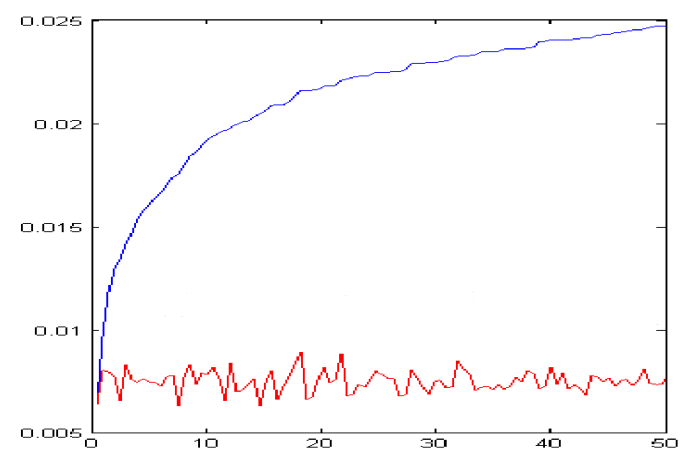

Figure 10: Mean error over a few hundred realisations for degenerate noise. Parameters: $\nu=1, \sigma=1 / \sqrt{15}, \varepsilon=0.1$. Small initial conditions of order $\varepsilon$.

\subsection{Higher order effects}

In [8] higher order corrections are studied for the solution of the stochastic Swift-Hohenberg Equation (13) with degenerate noise. Using the higher order corrections (see, Lemma 18 in [8]) in the averaging result for the fast OU-process, one obtains an additional semi-martingale term in the amplitude equation.

To approximate this martingale term path-wise, the proofs are restricted to the case of one-dimensional dominant space, i.e. $\operatorname{dim}(\mathcal{N})=1$. For higher dimensional spaces, one can obtain similar results. But then only weak convergence of the approximation is established, and no path-wise error bounds are available (cf. [8]). 
These approximation depends on the averaging of stochastic integrals over a fast OU-process (see Lemma 6.1 from [4]), which is based on the martingale representation theorem. So, for the example here we consider (13) with respect to Neumann boundary conditions on the interval $[0, \pi]$. The approximation result is:

Theorem 5. (higher order correction) Let $v$ be a solution of (13) in $H^{\alpha}$ for $\alpha \geq 0$ with degenerate noise and with the initial conditions $v(0)=a(0) \cos +\psi(0)$, where $\psi(0) \in S$, and $a$ is a solution of (9).

Then for all $p \geq 1, T_{0}>0$ and all $\kappa>0$ sufficiently small, there exists $C>0$ such that for $\|v(0)\|_{\alpha} \leq \delta_{\varepsilon}$ for some $\delta_{\varepsilon} \in\left(0, \varepsilon^{-\frac{1}{3} \kappa}\right)$ we have

$$
\mathbb{P}\left(\sup _{T \in\left[0, T_{0}\right]}\|v(T)-a(T) \cos -\varepsilon b(T) \cos -\mathcal{Q}(T)\|_{\alpha}>\varepsilon^{\frac{4}{3}-\kappa}\right) \leq C \varepsilon^{p},
$$

where $\mathcal{Q}(T)$ is defined in (18) and the higher order correction $b$ is the solution of

$$
d b=\left[\left(\nu-\frac{3}{2} \sigma^{2}\right) b-\frac{3}{4} a^{2} b\right] d T+\frac{3}{2} \sigma^{2} a d B
$$

with initial condition $b(0)=0$ and $B$ is a one-dimensional Brownian motion arising from a martingale representation theorem.

Related results in this direction are discussed by Roberts and Wang [23], nevertheless their setting is slightly different, and they use averaging techniques that do not lead to explicit error estimates. Their model is very similar, but they use a coupled system of a slow SDE and a fast SPDE. This is similar to our case, once (13) is split into the slow dominant modes and the infinitely many fast stable modes.

We tried to find an effect that is not present in the amplitude equation, but present in the SPDE. We tried the slow motion along the ring of stationary solutions for $a \in \mathbb{R}$ for a two-dimensional $\mathcal{N}$. These are not present in the deterministic amplitude equation, as the argument of $a \in \mathbb{C}$ cannot change. Nevertheless there is a possibility that this might occur for the first FourierMode of $v$. Although our numerical results indicate that this does not happen, despite the fact that the higher order correction (21) would not preserve the angle.
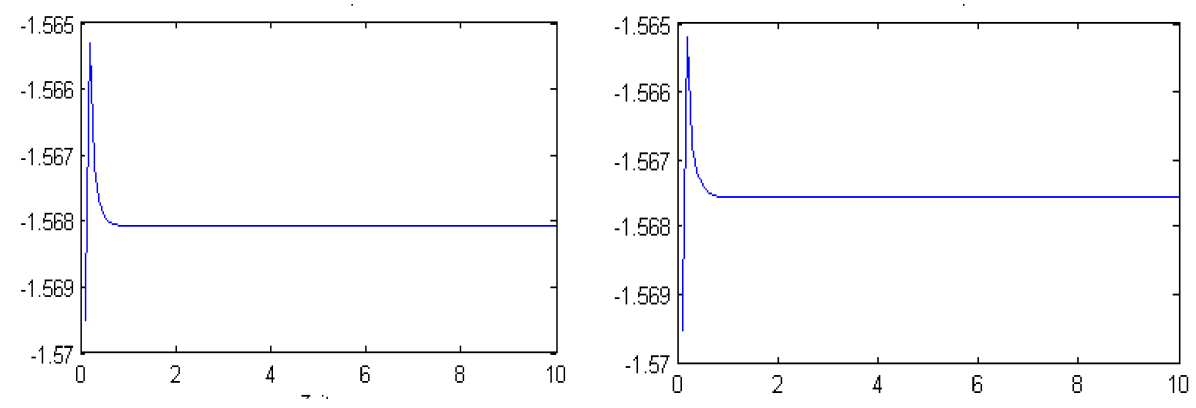

Figure 11: The argument of the first Fourier mode of $v$ does not move in time at all. Parameters: $\nu=1, \varepsilon=\frac{1}{10}$ and $\sigma^{2}=\frac{2}{3} \dot{0}, 99$ (left) and $\sigma^{2}=\frac{1}{3}$ (right) 


\section{Large domains}

In this section we briefly comment on some result about the stochastic SwiftHohenberg equation (13) on large or unbounded domain.

Consider as in [3] Equation (13) on a large domain $\left[\frac{-L}{\varepsilon}, \frac{L}{\varepsilon}\right]$ with additive homogeneous space-time noise of strength $\sigma \varepsilon^{3 / 2}$. They showed that, under appropriate scaling, its solutions can be approximated by the solution of the stochastic Ginzburg-Landau equation.

For (13) on the whole real line with degenerate additive noise, Axel Hutt and collaborators [16], [14] used a formal argument based on centre manifold theory. They showed that noise constant in space leads to a deterministic amplitude equation, which is stabilized by the impact of additive noise, while [9] made these results rigorous, at least for solutions decaying at infinity.

Here we state the result on the fast time-scale $t$ and the fast spacial scale $x$. The approximation result is:

Theorem 6. Let $u(t, x)$ be a solution of (3) on $\mathbb{R}$, with spacially constant noise, let $w_{A}$ be the formal approximation defined as

$$
w_{A}(T, X)=A(T, X) e^{i X / \varepsilon}+c . c,
$$

and $A(T, X)$ a solution of

$$
\partial_{T} A=4 \partial_{X}^{2} A+\left(\nu-\frac{3}{2} \sigma^{2}\right) A-3|A|^{2} A,
$$

such that $A \in C^{0}\left(\left[0, T_{0}\right], \mathcal{H}^{\alpha}\right)$ for $\alpha>\frac{1}{2}$, with initial condition

$$
\left\|u(0, \cdot)-\varepsilon A(0, \varepsilon \cdot) e^{i x}-\varepsilon \bar{A}(0, \varepsilon \cdot) e^{-i x}\right\|_{\infty} \leq d \varepsilon^{1-\kappa_{0}} \phi_{\varepsilon}
$$

for some fixed $d>0$ and for $\kappa_{0} \in(0,1)$. Then for each $T_{0}>0$ and $p>0$ there exist $C>0$, depending on $\sup _{\left[0, T_{0}\right]}\|A\|_{\alpha}$, such that

$$
\mathbb{P}\left(\sup _{t \in\left[0, \varepsilon^{-2} T_{0}\right]}\left\|u(t, x)-\varepsilon w_{A}\left(\varepsilon^{2} t, \varepsilon x\right)-\varepsilon \mathcal{Z}\left(\varepsilon^{2} t\right)\right\|_{\infty}>C \varepsilon^{1-\kappa_{0}} \phi_{\varepsilon}\right) \leq C \varepsilon^{p},
$$

where

$$
\phi_{\varepsilon}=\left\{\begin{array}{lll}
\varepsilon & \text { if } & \alpha>3 / 2, \\
\varepsilon \ln \left(\varepsilon^{-1}\right) & \text { if } & \alpha=3 / 2, \\
\varepsilon^{\alpha-\frac{1}{2}} & \text { if } & \alpha<3 / 2,
\end{array}\right.
$$

and $\mathcal{Z}(T)$ is the fast $O U$-process defined in (19).

In Figure 12 the show the mean and mean uniform error for various parameter regimes on the slow time-scale $T$. We computed the mean uniform error

$$
R_{1}(T)=\mathbb{E} \sup _{s \in[0, T]} \sup _{X \in[-1,1]}\left|\varepsilon^{-1} u\left(s \varepsilon^{-2}, X \varepsilon^{-1}\right)-w_{A}(s, X)-\mathcal{Z}(s, X)\right| .
$$

and the mean error

$$
R_{2}(T)=\mathbb{E} \sup _{X \in[-1,1]}\left|\varepsilon^{-1} u\left(T \varepsilon^{-2}, X \varepsilon^{-1}\right)-w_{A}(T, X)-\mathcal{Z}(T, X)\right| .
$$



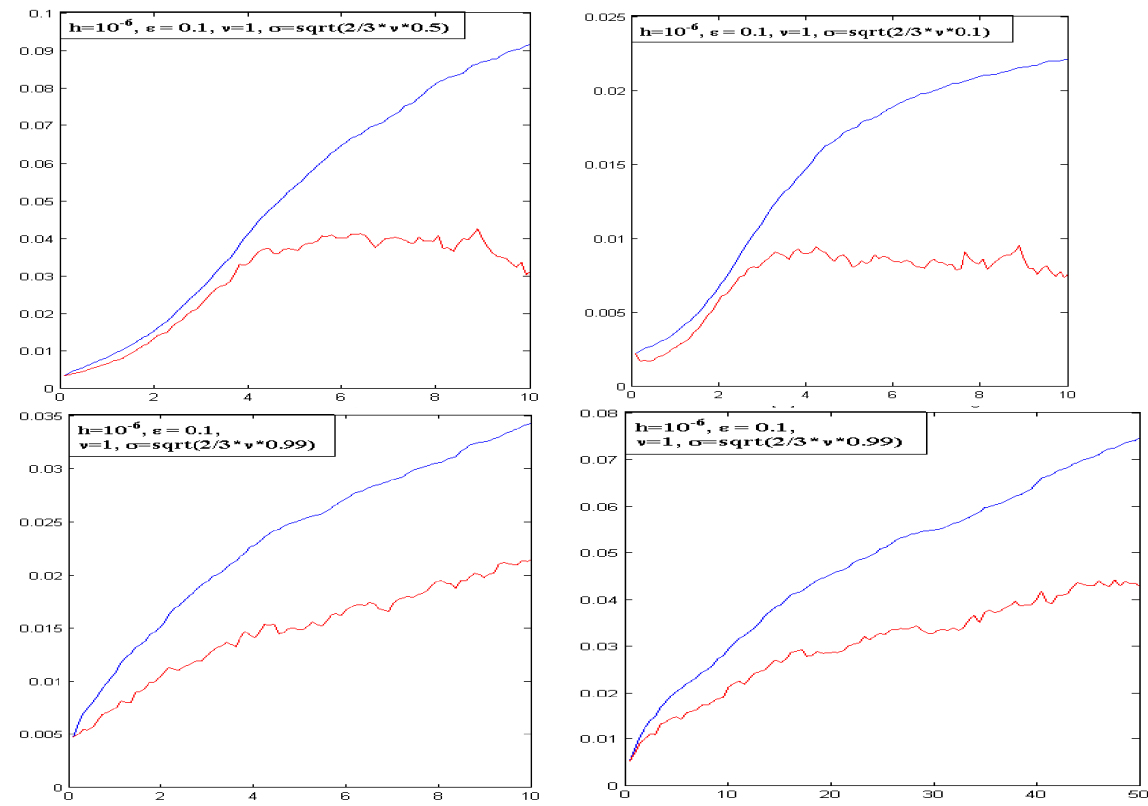

Figure 12: Various results for mean error and mean uniform error on large domains of order $\varepsilon^{-1}$ with periodic boundary conditions. We use the slow timescale $T=\varepsilon^{2} t$. The scaling with $\sigma$ is nicely seen in the first three pictures. The error bound is very good, even for large values of $T$.

\section{Acknowledgements}

Supported by Deutsche Forschungsgemeinschaft (DGF) "Multi-scale analysis of SPDEs" (DFG535-9/1). Wael W. Mohammed is supported by a fellowship from the Egyptian government in the Long Term Mission system.

\section{References}

[1] A. Abdulle, G. Pavliotis. Numerical Methods for Stochastic Partial Differential Equations with Multiple Scales. Preprint, (2011).

[2] D. Blömker, M. Hairer. Multiscale expansion of invariant measures for SPDEs. Commun. Math. Phys., 251(3):515-555 (2004).

[3] D. Blömker, M. Hairer, G. A. Pavliotis. Modulation equations: Stochastic bifurcation in large domains. Commun. Math. Phys.. Vol 258(2):479-512 (2005).

[4] D. Blömker, M. Hairer, G.A. Pavliotis. Multiscale analysis for stochastic partial differential equations with quadratic nonlinearities. Nonlinearity, 20:1-25 (2007).

[5] D. Blömker, A. Jentzen. Galerkin approximations for the stochastic Burgers equation, Preprint, (2009). 
[6] D. Blömker, S. Maier-Paape, G. Schneider. The stochastic Landau equation as an amplitude equation. Discrete Contin. Dyn. Syst. Ser. B, 1(4):527$541,(2001)$.

[7] D. Blömker, W.W. Mohammed. Amplitude equation for SPDEs with quadratic nonlinearities. Electronic Journal of Probability, 14:2527-2550 (2009).

[8] D. Blömker, W.W. Mohammed. Amplitude Equations for SPDEs with Cubic Nonlinearities. Preprint, (2010).

[9] D. Blömker, W.W. Mohammed. Modulation Equation for Stochastic SwiftHohenberg equation. Preprint, (2011).

[10] P. Collet, J.-P. Eckmann. The time dependent amplitude equation for the Swift-Hohenberg problem. Comm. Math. Phys., 132(1):139-153, (1990).

[11] M.C. Cross, P.C. Hohenberg. Pattern formation outside of equilibrium. Rev. Mod. Phys., 65:581-1112, (1993).

[12] G. Da Prato, J. Zabczyk. Stochastic equations in infinite dimensions, Cambridge University Press, Cambridge, (1992).

[13] W. E, D. Liu, E. Vanden-Eijnden. Analysis of multiscale methods for stochastic differential equations.Comm. Pure Appl. Math., 58(11):1544$1585,(2005)$.

[14] A. Hutt, A. Longtin, L. Schimansky-Geier. Additive global noise delays Turing bifurcations. Phys. Rev. Lett., 98:230601, (2007).

[15] A. Hutt, A. Longtin, L. Schimansky-Geier. Additive noise-induced Turing transitions in spatial systems with application to neural fields and the SwiftHohenberg equation. Physica D, 237:755-773, (2008).

[16] A. Hutt. Additive noise may change the stability of nonlinear systems. Europhys. Lett. 84(3):34003, (2008).

[17] A. Jentzen. Pathwise numerical approximation of SPDEs with additive noise under non-global Lipschitz coefficients. Potential Analysis, 31(4):375404, (2009).

[18] A. Jentzen, P. E. Kloeden, G. Winkel. Efficient simulation of nonlinear parabolic SPDEs with additive noise. The Annals of Applied Probability, 21(3):908-950, (2011).

[19] P. Kirrmann, G. Schneider, A. Mielke. The validity of modulation equations for extended systems with cubic nonlinearities. Proc. R. Soc. Edinb., Sect. A, 122(1-2):85-91, (1992).

[20] A. Mielke, G. Schneider. Attractors for modulation equations on unbounded domains - existence and comparison. Nonlinearity, 8(5):743-768, (1995).

[21] A. Mielke, G. Schneider, A. Ziegra. Comparison of inertial manifolds and application to modulated systems. Math. Nachr., 214:53-69, (2000). 
[22] A. J. Roberts. A step towards holistic discretisation of stochastic partial differential equations. ANZIAM J., 45(E):C1-C15, (2003).

[23] A. J. Roberts, Wei Wang. Macroscopic reduction for stochastic reactiondiffusion equations. Preprint, (2008), arXiv:0812.1837v1 [math-ph]

[24] G. Schneider. Error estimates for the Ginzburg-Landau approximation. $Z$. Angew. Math. Phys., 45(3):433-457, (1994).

[25] G. Schneider. The validity of generalized Ginzburg-Landau equations. Math. Methods Appl. Sci., 19(9):717-736, (1996). 